
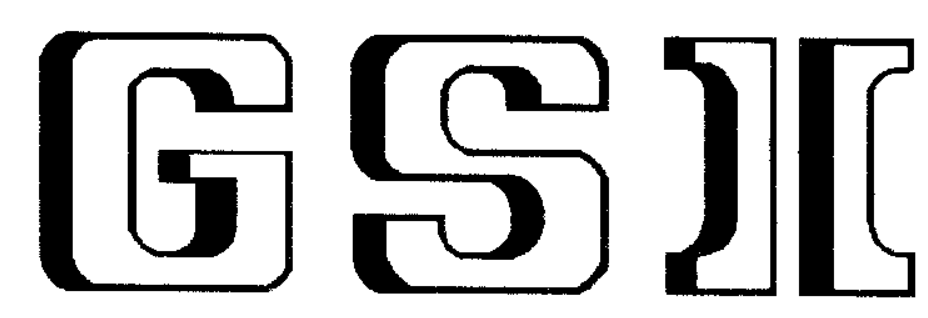

GSI-Preprint-99-07

Februar 1999

DEPOLARIZATION RADII OF LATENT HEAVY-ION TRACKS IN POLY(VINYLIDENE FLUORIDE)

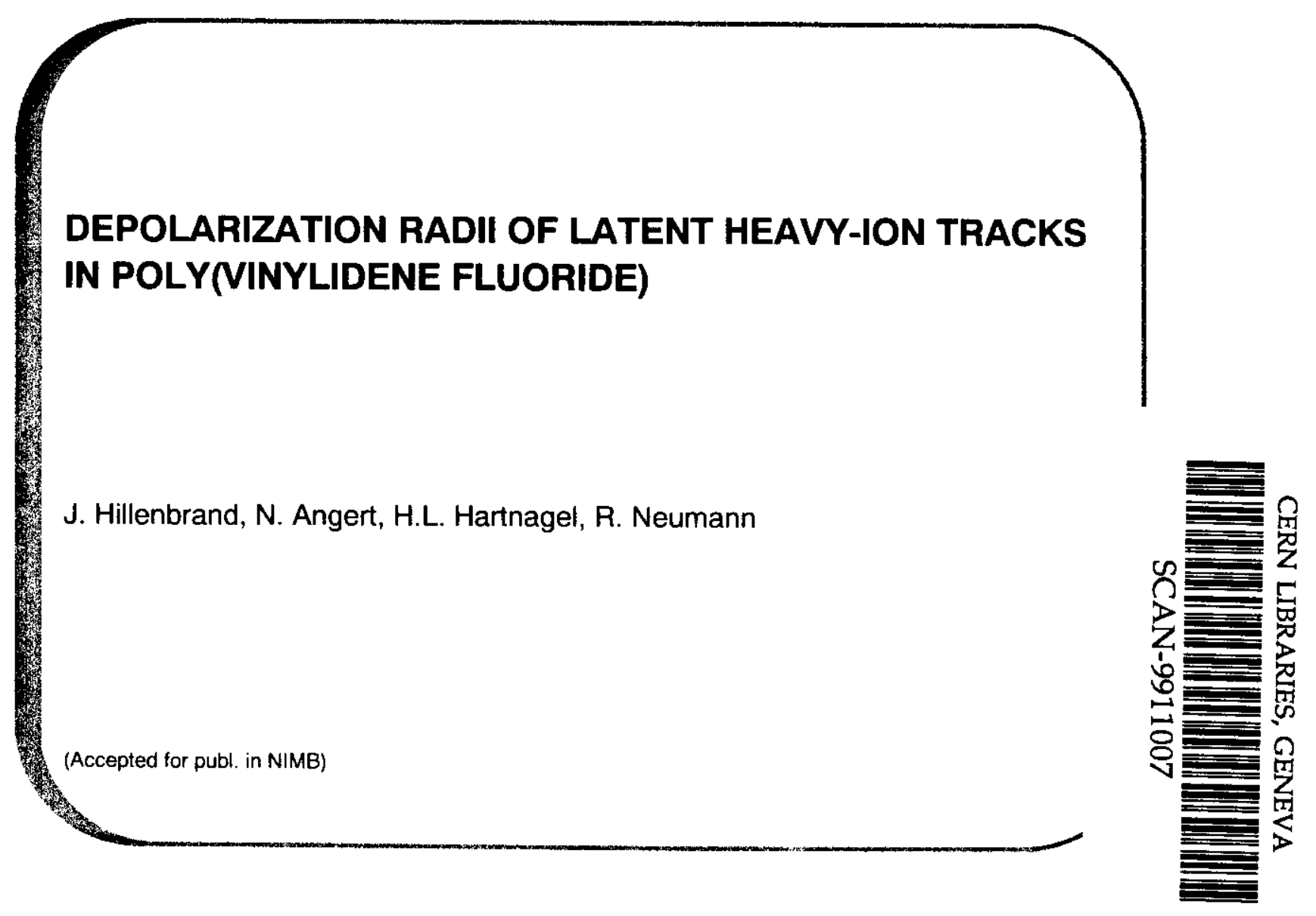

Gesellschaft für Schwerionentorschum mbH

Planckstraße + De4291 Damstedt " esmany

Postach 110652 . D-64200 Damistad - Cemany 


\title{
Depolarization radii of latent heavy-ion tracks in poly(vinylidene fluoride)
}

\author{
Hillenbrand ${ }^{\mathrm{a}}$, N. Angert ${ }^{\mathrm{a}}$, H.L. Hartnagel ${ }^{\mathrm{b}}$, R. Neumann $\mathrm{N}^{\mathrm{a*}}$ \\ ${ }^{a}$ Gesellschaft fiur Schwerionenforschung (GSI), Planckstr. 1, D-64291 Darmstadt, Germany

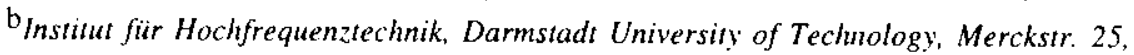 \\ D-64283 Darmstadt, Germany
}

* Now with Aist GmbH, D-84539 Ampfing, Germany

** Corresponding author: Tel. ++49615971 2172; e-mail: R.Neumann@gsi.de

\begin{abstract}
Poly(vinylidene fluoride) (PVDF) is a ferro- and piezoelectric material with a high piezoelectric constant, being of interest for numerous technical applications. The piezoelectric activity mainly originates from aligned molecular dipoles in the crystalline regions. In our work, foils of poled $\beta$-PVDF are exposed to heavy ions (with kinetic energies of about one to several $\mathrm{GeV}$ ), employing different ion species and fluences. Irradiation with energetic heavy ions reduces the crystallinity and in consequence also the remanent polarization. Adjacent irradiated and nonirradiated zones are provided by protecting part of each sample with a mask. A sinusoidal voltage applied to the foil causes a periodic thickness change on the nanometer scale whose size differs for irradiated and virgin areas. These oscillation amplitudes, monitored by line scans with a commercial profilometer, can be related to a mean depolarization radius of the damage trails of single ions. From a comparison with ion-track radii extracted from XRD and SAXS measurements we conclude that an ion track in poled PVDF consists of an amorphous core, surrounded by a concentric shell of still partially crystalline but depolarized material.
\end{abstract}

PACS: 07.10.Yg; 61.80.Jh; 61.82.Pv; 77.65.Bn

Keywords: Ferroelectric polymers; Piezoelectricity; Ion-induced depolarization 


\section{Introduction}

Poly(vinylidene fluoride) (PVDF) and its tri- and tetrafluorethylene copolymers are ferroelectric materials. This property which is unusual for polymers, was indicated for the first time in 1969 in the course of the discovery of the piezoelectric effect in poled PVDF [1]. Two years later, the proof of the pyroelectric effect [2] provided a further hint to the ferroelectric origin of both effects. Since then, numerous activities focused on this new class of materials, from the point of view of basic as well as applied research. Based on these specific properties, a series of technical applications were realized [3-7]. A few examples may suffice here: microphones [8], hydrophones [9], stereo headphones [10], sensors used for medical purposes [11], actuators [12], and infrared detectors [13].

The piezoelectric activity mainly originates from aligned molecular dipoles in the crystalline regions $[6,14]$. The alignment of the dipole moments is established during the poling process, leading to a remanent polarization $[13,15]$. PVDF is characterized by a linear relationship between this polarization and the macroscopic piezoelectric constant $d_{33}$. When irradiating a target of poled PVDF with energetic heavy ions, the projectiles transfer their kinetic energy to the material by interaction (as in any solid) with the target electrons, thereby producing along their trajectories continuous, long, and narrow damage trails of cylindrical shape. Within these so-called latent tracks with a diameter of the order of ten to several tens of $\mathrm{nm}$, the structure of PVDF is modified significantly in comparison with the intact surrounding material, since the energy loss of the ions induces the scission of bonds, the occurrence of radicals and of new kinds of bonds, and the dislocation of molecules or fractions of molecules. The structural damage, including the destruction of crystallinity, leads to a change of physical and chemical properties, in particular to a reduction of the polarization.

After exposure to energetic heavy ions, PVDF samples have been studied concerning the change of crystallinity [16,17] and the lowering of the Curie temperature [17-19]. The analytical methods applied to ion-irradiated PVDF include X-ray photon spectroscopy (XPS) [20-25], 
electron paramagnetic resonance (EPR) [22,24,26,27], infrared (IR) spectroscopy [21-26], and outgassing mass spectrometry [22,23].

The present paper reports the modification of the constant $d_{33}$ in poled $\beta$-PVDF through the irradiation by heavy ions with kinetic energies of the order of one to several GeV [28]. These projectiles have penetration depths up to about $150 \mu \mathrm{m}$. In irradiated target areas, $d_{33}$ exhibits a decrease whose size can be varied over a wide range as a function of the ion fluence. The decrease results from the reduction of the remanent polarization in a large number of single latent tracks. The values of $d_{33}$ in irradiated and in nonirradiated sample regions can be measured by monitoring the reciprocal piezoelectric effect as a function of the surface position. This is achieved by voltage-induced thickness oscillation and nanoprofilometry. The ratio of the oscillation amplitudes of virgin and ion-irradiated sample areas is a measure of depolarization. In a simplified approach, a mean ion track radius can be derived from this ratio, characterizing a microscopic cylindric volume, caused by a single heavy ion, in which the polarization is destroyed. We compare this depolarization radius with mean radii of latent ion tracks extracted from X-ray diffraction (XRD) and small-angle X-ray scattering (SAXS) measurements [16,29]. Ion track radii of a comparable size in PVDF have been recently determined also by FTIR measurements [30].

\section{Experimental arrangement and measurements}

This chapter gives only a brief outline of the experimental arrangement and data acquisition. A more detailed description of our apparatus and signal processing will be presented elsewhere [31].

All studies have been performed with PVDF foils (from Piezotech company) of thickness 25 $\mu \mathrm{m}$, covered on both sides with $100 \mathrm{~nm}$ thick aluminium layers. Stacks of four to five circular samples with a diameter $50 \mathrm{~mm}$ were irradiated normal to the sample surface by heavy ions from the Universal Linear Accelerator (UNILAC) of GSI. A part of the incident beam was blocked by a mask so that each sample consisted of an irradiated and a nonirradiated part. The 
pristine foil has an average piezoconstant $d_{33}=15 \mathrm{pC} / \mathrm{N}=15 \mathrm{~nm} / \mathrm{kV}$. By applying a voltage of $400 \mathrm{~V}$, the foil alters its thickress by $6 \mathrm{~nm}$. We did not use higher voltages in order to avoid polarization changes during the measurement. A sinusoidal voltage was applied to the PVDF samples and the amplitudes of the resulting periodic thickness changes were determined by surface profilometry [28].

Line scans were made electromechanically by moving the sample beneath the diamond-tipped stylus of a commercial surface profilometer (DEKTAK 8000 from Veeco Instruments). The lateral resolution is limited by the stylus diameter of $25 \mu \mathrm{m}$. This means that the instrument can provide only a macroscopic measurement of $d_{33}$. Fig. 1 shows a scan performed in $50 \mathrm{~s}$ on a nonirradiated foil, while applying to the foil a sinusoidal voltage of $U_{\mathrm{rms}}=272 \mathrm{~V}$ with a modulation frequency of $27.27 \mathrm{~Hz}$. The envelopes of the oscillation amplitudes of four scans over partly irradiated foils, achieved after filtering with a $1 \mathrm{~Hz}$ bandpass, are presented in Fig. 2. The region from 0 to about $290 \mu \mathrm{m}$ has been irradiated with a fluence of $2.5 \times 10^{10} \mathrm{Xe}$ ions $/ \mathrm{cm}^{2}$, while the zone from 290 to $600 \mu \mathrm{m}$ was protected against the ion beam by a mask. The oscillation amplitudes were reduced by the irradiation from roughly $A_{\text {ni }}$ (nonirradiated) $=8.5 \AA$ to about $A_{\mathrm{i}}$ (irradiated) $=7.5 \AA$. Similar measurements have been performed on PVDF foils irradiated with different kinds of ions and various fluences. Table 1 summarizes the kinetic energies of the ions just before entering the different foils of a stack and the mean electronic energy losses in the foils.

\section{Results and discussion}

As an example, Fig. 3 displays the ratio $A_{\mathrm{j}} / A_{\mathrm{ni}}=d_{33}$ (irradiated) $/ d_{33}$ (nonirradiated) versus the fluence for the case of irradiation with $\mathrm{Zn}$ ions. For each fluence value, the data points are extracted from the second foil of the respective sample stack. The solid curve fitted to the data represents a mean effective damage radius $R_{\text {eff }}$ as discussed subsequently. The dashed fit curves correspond to a $\pm 10 \%$ error bar of the value of $R_{\text {eff }}$ extracted from the solid curve. 
We use a model [28] based on the following assumptions: Each ion track consists of a cylindric damage volume with an effective radius $R_{\mathrm{eff}}$, in which the polarization is destroyed. All these microscopic track volumes sum up to a macroscopic fraction of the total volume of the ionirradiated PVDF sample. The ion-induced decrease of the average piezoelectric constant is proportional to this fraction. From these assumptions follows that the relation between the oscillation amplitudes $A_{\mathrm{ni}}$ and $A_{\mathrm{i}}$, averaged over macroscopic sample regions, and the microscopic depolarization radius $R_{\mathrm{eff}}$ of a single ion is described by

$$
A_{\mathrm{i}}=A_{\mathrm{ni}} \cdot \exp \left(-\pi R_{\mathrm{eff}^{2}}^{2} F\right)
$$

where $F$ is the ion fluence. Such radii for different $\mathrm{d} E / \mathrm{d} x$ ion energy losses determined with the above equation are plotted in Fig. 4. The ion species employed for different groups of data points are given in the diagram. The data points of such a group result from the measurements at a single foil stack, the foils in a stack being numbered along the ion trajectory. Each data point relates to one specific foil as indicated by numbers. Each value is provided with a small and a large vertical error bar. The small error originates only from the measurement of the piezoelectric effect, while the larger one contains in addition the uncertainty of the fluence measurement. The $\mathrm{d} E / \mathrm{d} x$ abscissa values of all radii have been determined by means of the TRIM code [32]. For the sake of clarity, their error bars of approximately $\pm 15 \%$ have been omitted in Fig. 4.

The set of data points plotted in Fig. 4 is compatible with a $(\mathrm{d} E / \mathrm{d} x)^{1 / 2}$ dependence of the depolarization radius, in accordance with model assumptions on the radial energy deposition in heavy-ion tracks $[33,34]$. Nevertheless, Fig. 4 gives rise to briefly comment the problem that the effective radii depend not only on the energy loss, but also on the absolute kinetic energy and thus on the velocity of the ions. This velocity effect has been studied intensively for insulators [35, and references therein]. As mentioned above, the data illustrated in Fig. 4 consists of five groups, each group representing one of the ion species $\mathrm{Zn}, \mathrm{Se}, \mathrm{Xe}, \mathrm{Au}$, and $\mathrm{U}$. With increasing foil number, that means, with decreasing projectile velocity, the radii of such a 
group grow more strongly than expected for a $(\mathrm{d} E / \mathrm{d} x)^{1 / 2}$ dependence in the case of a pure energy loss effect. In view of the size of the error bars, one should not overstress the significance of the velocity effect in the present work. However, when considering the data points of a single ion species, the fluence uncertainty is ignorable, since each foil of the stack was exposed to the same fluence. Thus, the significance of the velocity effect can be judged with reference to the error bar attributed to the measurement of the piezoelectric effect.

For all $\mathrm{d} E / \mathrm{d} x$ values the depolarization radii are about 1.5 times larger than radii gained by XRD [16] and by SAXS [29] measurements. By evaluating the decrease of the XRD reflex intensity, an average radius is attributed to the track zones in which the crystallinity is destroyed. In contrast to that, SAXS is sensitive to a density reduction produced along the ion trajectory in crystatline as well as in amorphous polymer regions. Therefore, a mean radius derived from a SAXS pattern represents the full tracks encompassing both crystalline and amorphous zones. In conclusion, the difference in size between the depolarization radii and those obtained by XRD and SAXS gives rise to the assumption that an ion track in poled PVDF consists of an amorphous core surrounded by a cylindric shell of still partially crystalline but depolarized material.

\section{Summary}

Locally resolved measurements of the piezoelectric constant $d_{33}$ of poled $\beta$-PVDF foils have been performed after irradiation by energetic heavy ions. The data acquisition technique employed the reciprocal piezoelectric effect and required to register oscillation amplitudes and changes of these amplitudes on the nanometer scale. Irradiated as well as virgin areas were provided on each sample by masking part of the target against the ion beam. Applying a sinusoidal voltage to the PVDF foils, the periodic thickness changes were monitored by line scans with a diamond-tipped stylus of a commercial nanoprofilometer. This allowed us to determine $d_{33}$ of irradiated and of nonirradiated material by means of single line scans. 
The ion-induced decrease of the oscillation amplitude is a measure of that fraction of the foil volume, in which the polarization has been destroyed. Supposing that this volume fraction is the sum of all microscopic cylindric ion track volumes, an effective depolarization radius $R_{\mathrm{eff}}$ of these tracks can be derived. From the comparison with mean track radii, determined by XRD and SAXS, we conclude that the depolarization radius represents an ion track with an amorphous core and a concentric shell, in which the polymer is still partially crystalline but completely depolarized.

\section{References}

[1] H. Kawai, Jpn. J. Appl. Phys. 8 (1969) 975.

[2] J.G. Bergman, J.H. McFee and G.R. Crane, Appl. Phys. Lett. 18 (1971) 203.

[3] G.M. Sessler, J. Acoust. Soc. Am. 70 (1981) 1596.

[4] G.M. Sessler, Electrets, Topics in Appl. Physics, Vol. 33, Springer, Berlin, 1987.

[5] T.T. Wang, J.M. Herbert, and A.M. Glass (Eds.), The applications of ferroelectric polymers, Blackie, Glasgow, 1988.

[6] G. Eberle, H. Schmidt and W. Eisenmenger, IEEE Trans. Dielectr. Electr. Ins. 3 (1996) 624.

[7] S. Bauer and S.B. Lang, IEEE Trans. Dielectr. Electr. Ins. 3 (1996) 647.

[8] R. Schellin, G. Heß, W. Kühnel, G.M. Sessler and E. Fukada, IEEE Trans. Electr. Ins. 27 (1992) 867.

[9] B. Woodward, Acoustica 37 (1977) 264.

[10] F. Micheron, P. Ravinet, D. Guillou and C. Claudepierre, Ferroelectrics 51 (1983) 143.

[11] S.B. Lang, B.D. Sollish, M. Moshitzhy and E.H. Frei, Ferroelectrics 24 (1980) 289.

[12] V.H. Schmitt, M. Klakken and H. Darejeh, Ferroelectrics 51 (1983) 105.

[13] H. Nalwa, Ferroelectric Polymers, Marcel Dekker (1995). 
[14] M. Sessler, D.K. Das-Gupta, A.S. DeReggi, W. Eisenmenger, T.Furukawa, A. Giacometti and R. Gerhart-Multhaupt: IEEE Trans. Electr. Ins. 27 (1992) 872. [15] T. Furukawa, IEEE Trans. Electr. Ins. 24 (1989) 375.

[16] V. Chailley, E. Balanzat and E. Dooryhée, Nucl. Instr. and Meth. B 105 (1995) 110.

[17] E. Petersohn, N. Betz and A. Le Moël, Nucl. Instr. and Meth. B 105 (1995) 267.

[18] D. Schlößer and A. Le Moël, Nucl. Instr. and Meth. B 105 (1995) 278.

[19] E. Petersohn, N. Betz and A. Le Moël, Nucl. Instr. and Meth. B 107 (1996) 369.

[20] A. Le Moël, J.P. Duraud and E. Balanzat, Nucl. Instr. and Meth. B 18 (1986) 59.

[21] A. Le Moël, J.P. Duraud, C. Lecomte, M.T. Valin, M. Henriot and C. Le Gressus, Nucl. Instr. and Meth. B 32 (1988) 115.

[22] A. Fina, A. Le Moël, J.P. Duraud and M.T. Valin, Nucl. Instr. and Meth. B 42 (1989) 69.

[23] J.P. Duraud, A. Le Moël, N. Betz, A. Fina and E. Balanzat, Radiat. Eff. Def. Solids 110 (1989) 33.

[24] N. Betz, A. Le Moël, J.P. Duraud, E. Balanzat and C. Darnez, Macromolecules 25 (1992) 213.

[25] L. Calgagno, P. Musumeci, R. Percolla and G. Foti, Nucl. Instr. and Meth. B 91 (1994) 461.

[26] E. Balanzat, S. Bouffard and A. Le Moël, Nucl. Instr. and Meth. B 91 (1994) 140.

[27] N. Betz, E. Petersohn and A. Le Moël, Nucl. Instr. and Meth. B 116 (1996) 207.

[28] J. Hillenbrand, Doctoral Thesis, Darmstadt University of Technology (1997).

[29] T. Steckenreiter, Doctoral Thesis, Darmstadt University of Technology (1997).

[30] A. Le Bouëdec, N. Betz, S. Esnouf and A. Le Moël, Nucl. Instr. and Meth. B, these Proceedings.

[31] J. Hillenbrand and R. Neumann, to be published.

[32] J.F. Ziegler, J. P. Biersack and U. Littmark, The Stopping and Ranges of Ions in Solids (Pergamon, Oxford, 1985).

[33] R. Katz and E.J. Kobetich, Phys. Rev. 170 (1968) 401. 
[34] M. Krämer, Nucl. Instr. and Meth. B 105 (1995) 14.

[35] A. Meftah, F. Brisard, J.M. Costantini, M. Hage-Ali, J.P. Stoquert, F. Studer and M.Toulemonde, Phys. Rev. B 48 (1993) 920.

Table 1

\begin{tabular}{|c|c|c|c|c|c|c|c|c|c|c|}
\hline \multirow[b]{2}{*}{ Ion } & \multicolumn{2}{|c|}{ Foil \#1 } & \multicolumn{2}{|c|}{ Foil \#2 } & \multicolumn{2}{|c|}{ Foil \#3 } & \multicolumn{2}{|c|}{ Foil \#4 } & \multicolumn{2}{|c|}{ Foil \#5 } \\
\hline & $E$ & $\mathrm{dE} / \mathrm{dx}$ & $\mathrm{E}$ & $\mathrm{dE} / \mathrm{dx}$ & E & $\mathrm{dE} / \mathrm{dx}$ & E & $\mathrm{dE} / \mathrm{dx}$ & E & $\mathrm{dE} / \mathrm{dx}$ \\
\hline $\mathrm{Zn}$ & 11.4 & 4.5 & 9.8 & 4.9 & 8.0 & - & 6.1 & 6.2 & - & - \\
\hline $\mathrm{Se}$ & 11.4 & 5.6 & 9.7 & 6.0 & 7.8 & 6.7 & 5.8 & 7.6 & 3.5 & 8.4 \\
\hline $\mathrm{Xe}$ & 11.4 & 10.9 & 9.2 & 11.6 & 7.0 & - & 4.5 & 13.7 & - & - \\
\hline $\mathrm{Au}$ & 11.6 & 18.0 & 9.2 & 18.3 & 6.9 & - & 4.3 & 19.3 & 0.2 & - \\
\hline $\mathrm{U}$ & 11.4 & 21.5 & 9.1 & - & 6.8 & 22.9 & 4.4 & 22.2 & 2.0 & 17.5 \\
\hline
\end{tabular}

Figure and Table Captions

Fig. 1. Raw data of a scan performed on a nonirradiated PVDF foil, a sinusoidal voltage of $U_{\text {eff }}=272 \mathrm{~V}$ at a frequency of $\sim 27 \mathrm{~Hz}$ being applied to the sample during the measurement.

Fig. 2. Thickness oscillation amplitudes achieved by filtering of the raw data with a $1 \mathrm{~Hz}$ bandpass. The four scans have been performed with a lateral displacement of $16.7 \mu \mathrm{m}$ from one to the next. The surface region from 0 to about $290 \mu \mathrm{m}$ was irradiated with a fluence of $2.5 \times 10^{10} \mathrm{Xe}$ ions $/ \mathrm{cm}^{2}$, whereas the remaining surface was protected against the ion beam.

Fig. 3. Ratio of oscillation amplitudes versus ion fluence. The samples were irradiated with $\mathrm{Zn}$ ions. Each open circle represents a data point resulting from a measurement at the second foil of a PVDF foil stack. The respective mean of each set of these data points is given by a full 
circle. The average electronic energy loss in the foils was $\mathrm{d} E / \mathrm{d} x=4.9 \mathrm{keV} / \mathrm{nm}$. The solid curve represents a mean effective damage radius $R_{\mathrm{eff}}$. The value of $R_{\mathrm{eff}}$ deduced from the solid curve shifts by $\pm 10 \%$ when using the dashed fit curves.

Fig. 4. Mean effective depolarization radius vs. electronic ion energy loss. Each group of data points relates to a different ion species as indicated (the open circles belonging to $\mathrm{Zn}$ ), and results from measurements at a single foil stack. The foils in a stack are numbered along the direction of ion propagation. Each data point originates from one specific foil as expressed by the numbers. The small error bars originate only from the measurement of the piezoelectric effect, the large ones include also the fluence uncertainy.

Table 1. Compilation of the kinetic energies $E(\mathrm{MeV} / \mathrm{u})$ of the ions before entering the foils and of the mean electronic energy losses $\mathrm{d} E / \mathrm{d} x(\mathrm{keV} / \mathrm{nm})$ in the foils. Missing values indicate that the ions did not reach the foil or that no experimental data was acquired. 


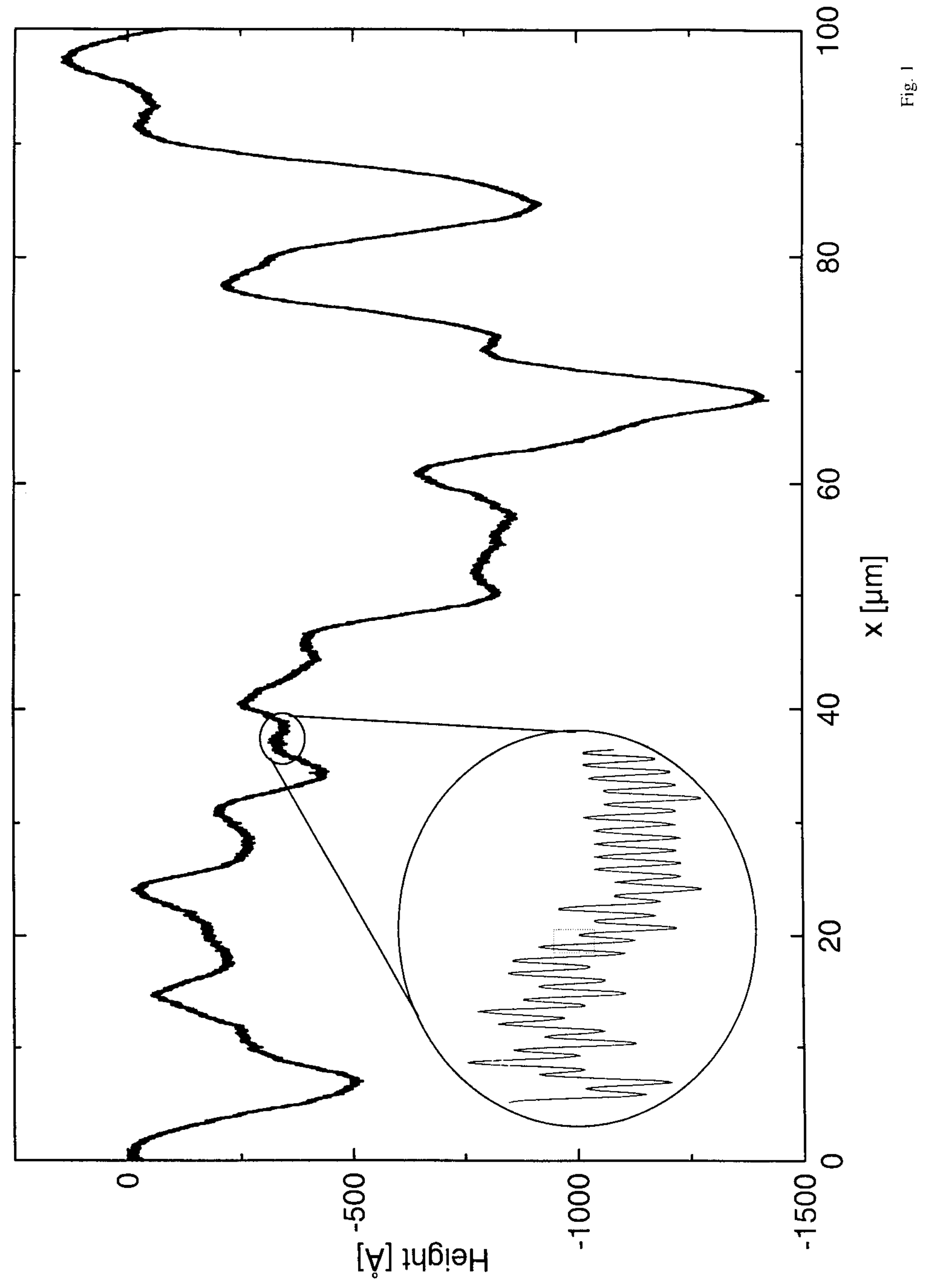




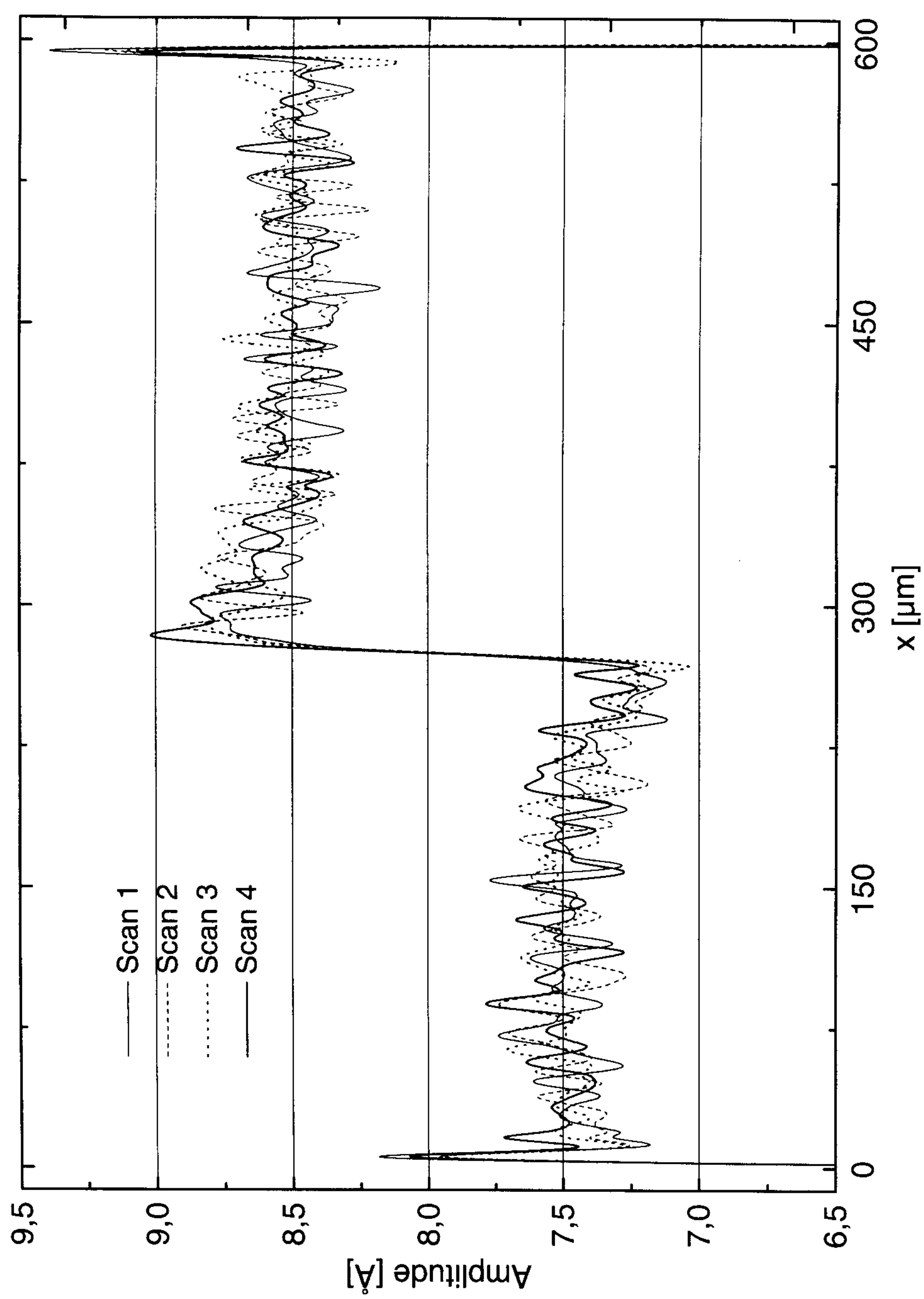




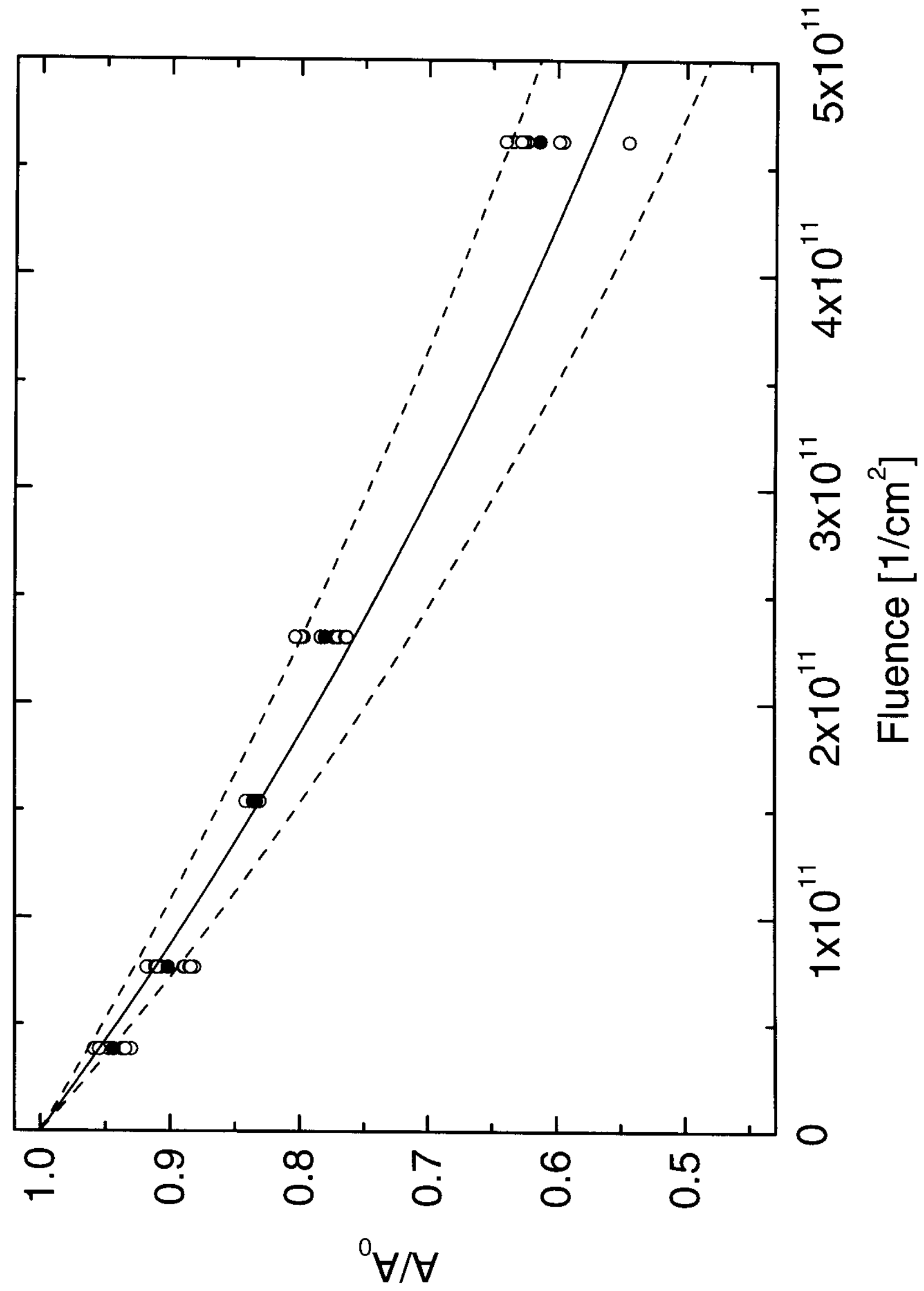




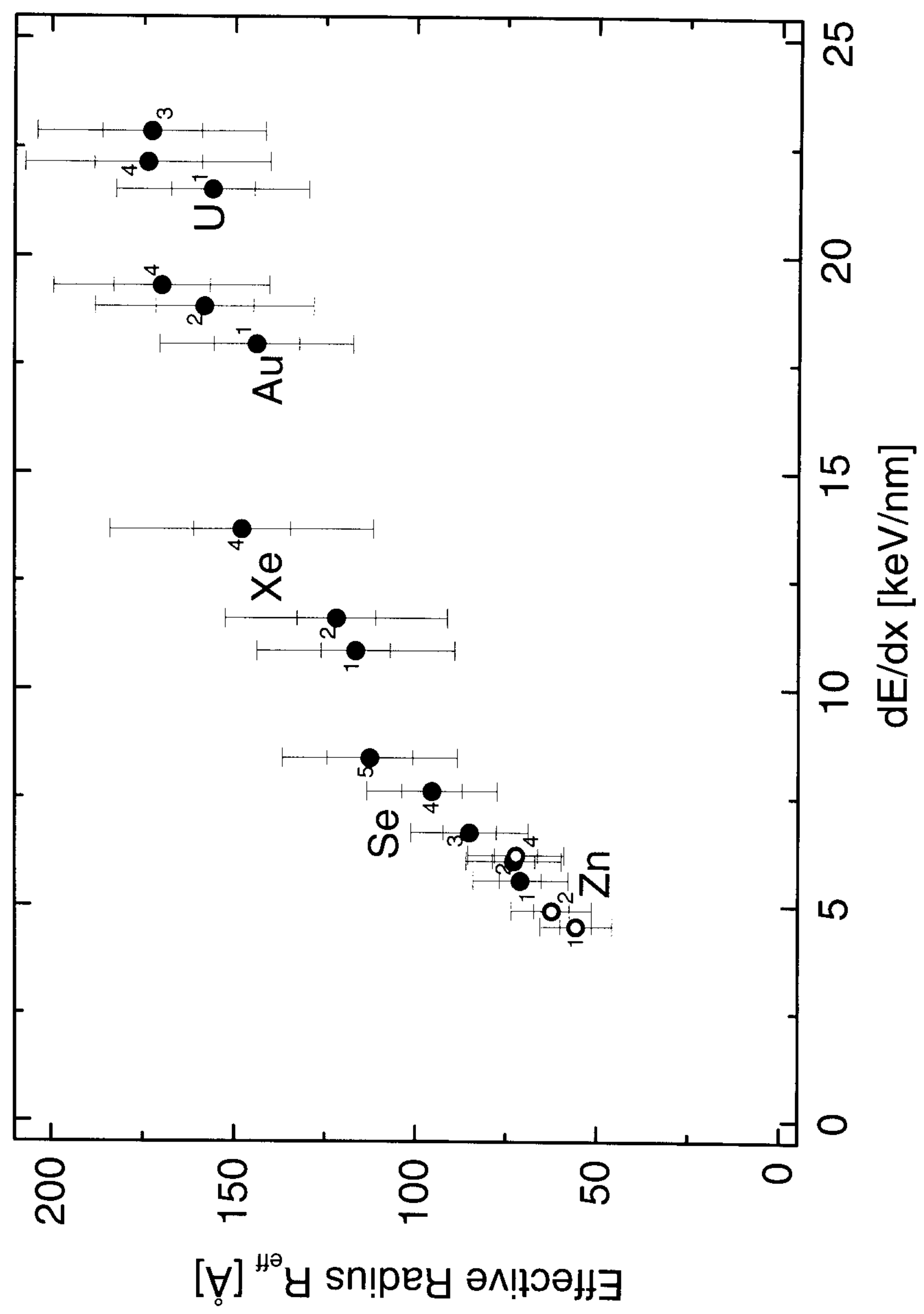

